\title{
A SARS-CoV-2 Prophylactic and Treatment; A Counter Argument Against The Sole Use of Chloroquine
}

\author{
Markus Depfenhart ${ }^{1 *}$, Gottfried Lemperle ${ }^{2}$, Markus Meyer $^{3}$, Marina Rautenbach $^{4}$, Dario Bertossi ${ }^{5}$ \\ and Danielle de Villiers ${ }^{6}$ \\ ${ }^{1}$ Faculty of Medicine, Venlo University BV, Netherlands
}

${ }^{2}$ Division of Plastic Surgery, University of California, USA

${ }^{3}$ Faculty of Medicine, Universitas Indonesia, Indonesia

${ }^{4}$ Faculty of Science, University of Stellenbosch, South Africa

${ }^{5}$ Maxillofacial Plastic Surgery Unit, University of Verona, Italy

${ }^{6}$ Research and Development, Medika SA, South Africa

*Corresponding author: Markus Depfenhart, Faculty of Medicine, Venlo University B.V, Venlo, Netherlands.

To Cite This Article: Depfenhart M, Gottfried L, Markus M, Marina R, Dario B, Danielle de V. A SARS-CoV-2 Prophylactic and Treatment; A Counter Argument Against The Sole Use of Chloroquine. 2020 - 8(4). AJBSR.MS.ID.001283. DOI: 10.34297/AJBSR.2020.08.001283.

Received: 畊 April 01, 2020; Published: 制 April 09, 2020

\begin{abstract}
A better knowledge of the SARS-CoV-2 virus and its underlying pathobiology is accumulating every day. Of huge importance now is to provide a fast, cost effective, safe, and immediately available pharmaceutical solution to curb the rapid global spread of SARS-CoV-2. This Opinion discusses the demands for such an ideal drug and taking into account an aspect of viral mechanisms of infection. An effective prophylactic medication to prevent viral entry has to contain, at least, either a TMPRSS2 inhibitor or a competitive virus ACE2 binding inhibitor. Using bromhexine at a dosage that selectively inhibits TMPRSS2 and, in so doing, inhibits TMPRSS2-specific viral entry is likely to be effective against SARS-CoV-2. We propose the use of bromhexine as a prophylactic and treatment. We encourage the scientific community to assess bromhexine clinically as a prophylactic and curative treatment. If proven to be effective, this would allow a rapid, accessible and cost-effective application worldwide.
\end{abstract}

Keywords: SARS-CoV-2; COVID-19; Coronavirus; Prophylactic; Treatment; Anti-viral drugs; Drug combinations; Bromhexine

\section{Introduction}

As the world witnesses the alarming levels of spread and severity of atypical pneumonia COVID-19, strategies to combat this outbreak are in dire need. The first sequence of SARS-CoV-2 was published online one day after its confirmation on behalf of Zhang and colleagues [1]. SARS-CoV-2 sequences isolated from all over the world have now been deposited in gene banks [2,3]. Sharing more genome sequences of the newly emerging SARSCoV-2 allows analysis of this new coronavirus (CoV), improving phylogenetic analysis and, most important, recognizing mutations between differing strains. Identifying the closest viral relatives of SARS-CoV-2 is greatly assisting studies of viral function. Ultimately, this gives rise to the understanding of what is unique and what is conserved in this new SARS-CoV-2 virus; be it structure, its host cell attachment and entry, or replication, making it possible to identify treatment targets. Currently, the treatment is mainly symptomatic and supportive care. Tremendous efforts have been undertaken and large amounts of money have been invested in vaccine development against influenza-type viruses. There are approximately 40 companies in advanced stages of vaccine development [4]. Disadvantages with cutting-edge vaccines are that they take months to years to develop and to approve, and they become obsolete if the virus evolves. There are already a number of reviews on potential treatment strategies against COVID-19 [57]. Drug repurposing is an attractive alternative drug discovery strategy in this time of urgency. 


\section{Proposed Treatment Strategy Against COVID-19}

The first step in CoV infection is the interaction of host cells with the viral envelope Spike (S) glycoprotein.SARS-CoV-2 employs two routes for host cell entry, which are dependent on the localization of the proteases required for activation of the $S$ protein [8]. Binding of SARS-CoV-2 to the cellular receptor, angiotensin converting enzyme 2 (ACE2), can result in uptake of virions into endosomes, where the $\mathrm{S}$ protein is activated by the $\mathrm{pH}$-dependent cysteine protease cathepsin B and L (cathepsin B/L) [9-11]. Alternatively, the spike protein can be activated by the serine protease TMPRSS2, resulting in fusion of the viral membrane with the plasma membrane [12]. Seeing as the S protein has pivotal roles in viral infection [13] we propose interfering with the $\mathrm{S}$ protein activation and hence viral pathogenesis.

Recently, publications on COVID-19 have brought attention to the possible benefit of repurposing the drug chloroquine in the treatment of patients infected by SARS-CoV-2 [14,15]. Chloroquine (N4-(7-Chloro-4-quinolinyl)-N1,N1-diethyl-1,4-pentanediamine), an FDA-approved drug [16], has been used to treat malaria and amebiasis for many years [17], as well as autoimmune diseases. Viral fusion and release of the genetic components is highly dependent on the endosomal pathway and particularly $\mathrm{pH}$. Chloroquine can affect virus infection in many ways. Of particular importance is that Chloroquine is known to block virus infection by increasing endosomal $\mathrm{pH}$ required for virus/endosome fusion [18] and release of viral RNA into the cytosol. Past research on chloroquine has shown in vitro activity against many different viruses, but no benefit in animal models [19]. Chloroquine in almost all animal models of different viral infections only partially worked or didn't work [20-22]. Treatment with chloroquine did not prevent influenza infection in a randomized, double-blind, placebo-controlled clinical trial.23 Conversely, it worked very well in vitro [24-26]. This could indicate that the main mechanism of action of chloroquine, in vivo, is via interference with the unspecific endosomal pathway. The extracellular concentration of the orally applied chloroquine, especially in lung tissue, in vivo, may not be high enough to inhibit virus binding via glycosylation of the binding pocket [18]. After the viral infection has spread in the body and due to the incredibly high viral loads, the unspecific pathway is mainly used for further virus replication. This may explain the recent success reported with chloroquine to assist in the curing of the virus. Whether chloroquine can treat COVID-19 alone and also work as a prophylactic is doubtful. This needs to be further investigated before masses of people start to take this relatively toxic drug as a preventive measure.

Inhibition of the serine protease, TMPRSS2, activity is an excellent target for antiviral intervention. Hoffman et al. [27] suggested that TMPRSS2 could be a potential therapeutic target for COVID-19 since entry of the virus into cells was reduced by camostat mesilate, a non-selective TMPRSS2 inhibitor. Non-selective inhibitors have greater, more severe side effects than selective inhibitors and currently camostat mesilate is only approved for treatment of chronic pancreatitis $[28,29]$ in Japan. Unfortunately, the drug is costly and won't be available to treat large-scale patient numbers.

TMPRSS2 is expressed highly in localized high-grade prostate cancers and in the majority of human prostate cancer metastases. Lucas et al. [30] showed a decrease in the frequency of metastases and a slowdown of the spread of metastases in mice with prostate cancer by using TMPRSS2 inhibitors. In particular, they identified bromhexine, an FDA approved ingredient [30] in mucolytic cough suppressants, as a potential TMPRSS2 inhibitor for their application. Bromhexine is orally readily bio-available.. Endonasal application is also a good alternative option. Bromhexine is an over-the-counter (OTC) drug [31] that is affordable with proven safety. Typically bromide compounds, especially aromatic bromide compounds, show a relatively high binding affinity for serine-containing peptide sequences, proteins and enzymes [30,32] Lucas et al. [30] show that this effect is due to a selective inhibition of TMPRSS2 by bromhexine. The available data suggests further that ambroxol, a metabolite of bromhexine, is a potent inducer of surfactant synthesis in AT2 cells [33-35]. Its lung protective properties have been discussed in infants and severely ill adult patients as well as the potential as an adjuvant in anti-infective therapy [34]. Thus, bromhexine also provides indirect protective effects. Laporte and colleague, Naesens [36], reported that bromhexine did not show any significant cell entry or replication inhibition effect in vitro in Influenza viruses. However, the authors showed that Influenza viruses utilize, contrary to SARS-CoV-2, a different extracellular host protease for priming. Thus, these results are not representative for SARS-CoV-2 [36].

In already infected individuals we believe it is essential to combine the lesser toxic chloroquine-derivate, hydroxyl chloroquine, with a TMPRSS2 inhibitor, like bromhexine, to block complete entry of the virus into host cells. In the case of prophylaxis the inhibition of the TMPRSS2 is essential [27] and the non-specific endosomal entry is negligible. An effective prophylactic medication to prevent viral entry has to contain, at least, either a TMPRSS2 inhibitor or a competitive virus ACE2 binding inhibitor. This will prevent further spreading of the virus through the host's body.

\section{Conclusion}

A prophylaxis strategy and a suitable treatment for the emerging SARS-CoV-2 is crucial for reducing the mortality and morbidity of this disease but developing and obtaining regulatory approval for new drugs can take years and is discordant with the urgent need for a therapy. Drug repurposing is an attractive alternative drug discovery strategy because there is the advantage of ease of 
access, decreased cost of development (as they have established manufacturing arrangements), and the possibility to provide a wide array of options for combination studies. The background pharmacological knowledge available for such compounds may also reduce concerns regarding adverse effects in patients as they have gone through rigorous safety and risk testing and are already approved as safe for human use. Using bromhexine at a dosage that selectively inhibits TMPRSS2 and, in so doing, inhibits TMPRSS2-specific viral entry is likely to be effective against SARS$\mathrm{CoV}-2$. We propose the use of bromhexine as a prophylactic and treatment. Furthermore, a combination with hydroxyl chloroquine, that is (amongst other functions) an effective endosomal protease inhibitor, inhibiting cathepsin B/L, could be a favorable combination for the treatment of moderate to severe COVID-19 cases. This combination would block virus-host cell entry completely by blocking the specific receptor mediated entry (via bromhexine) and endocytotic virus entry (via hydroxychloroquine sulfate). We can only encourage the scientific community to test bromhexine and the combination with hydroxychloroquine and to follow our recommended approach in order to also identify further ideal repurposing candidates according to the herein proposed criteria.

\section{Conflict of Interests}

The authors have declared that no conflicts of interest exist.

\section{References}

1. (2020) Novel 2019 Coronavirus Genome.

2. (2020) GSAID Database. Coronavirus.

3. SARS-CoV-2 (Severe acute respiratory syndrome coronavirus 2) Sequences. GenBank.

4. (2020) Covid-19 Vaccine Tracker. Regulatory Affairs Professionals Society.

5. Dyall J, Gross R, Kindrachuk J, Johnson RF, Olinger GG Jr, et al. (2017) Middle East Respiratory Syndrome and Severe Acute Respiratory Syndrome: Current Therapeutic Options and Potential Targets for Novel Therapies. Drugs 77(18): 1935-1966.

6. Zumla A, Hui DS, Azhar EI, Memish ZA, Maeurer M (2020) Reducing mortality from 2019-nCoV: host-directed therapies should be an option. The Lancet 395(10224): e35-e36.

7. Stebbing J, Phelan A, Griffin I, Catherine Tucker, Olly Oechsle, et al. (2020) COVID-19: combining antiviral and anti-inflammatory treatments. The Lancet Infectious Diseases 20(4): 400-402.

8. Simmons G, Zmora P, Gierer S, Heurich A, Pöhlmann S. Proteolytic activation of the SARS-coronavirus spike protein: Cutting enzymes at the cutting edge of antiviral research. Antiviral Research 100(3): 605-614.

9. Simmons G, Gosalia DN, Rennekamp AJ, Reeves JD, Diamond SL, et al. (2005) Inhibitors of cathepsin L prevent severe acute respiratory syndrome coronavirus entry. Proceedings of the National Academy of Sciences of the United States of America 102(33): 11876-1181.

10. Rubio Aliaga I, Frey I, Boll M, David A Groneberg, Hans M Eichinger, et al. (2003) Targeted disruption of the peptide transporter Pept2 gene in mice defines its physiological role in the kidney. Molecular and cellular biology 23(9): 3247-3252.

11. Ding N, Zhao K, Lan Y, Zi Li, Xioling Lv, et al. (2017) Induction of Atypical Autophagy by Porcine Hemagglutinating Encephalomyelitis Virus
Contributes to Viral Replication. Frontiers in cellular and infection microbiology $7: 56$.

12. Belouzard S, Millet JK, Licitra BN, Whittaker GR (2012) Mechanisms of coronavirus cell entry mediated by the viral spike protein. Viruses $4(6)$ : 1011-1133.

13. Hofmann H, Hattermann K, Marzi A, Gramberg T, Geier M, et al. (2004) $S$ protein of severe acute respiratory syndrome-associated coronavirus mediates entry into hepatoma cell lines and is targeted by neutralizing antibodies in infected patients. J Virol 78(12): 6134-6142.

14. Colson P, Rolain J M, Raoult D (2020) Chloroquine for the 2019 novel coronavirus SARS-CoV-2. International Journal of Antimicrobial Agents 55(3): 105923.

15. Gao J, Tian Z, Yang X (2020) Breakthrough: Chloroquine phosphate has shown apparent efficacy in treatment of COVID-19 associated pneumonia in clinical studies. Biosci Trends 14(1): 72-73.

16. Chloroquine Phosphate, Usp.

17. Aguiar ACC, Murce E, Cortopassi WA, Andre S Pimentel, Maria MFS Almeida, et al. (2018) Chloroquine analogs as antimalarial candidates with potent in vitro and in vivo activity. International journal for parasitology Drugs and drug resistance 8(3): 459-464.

18. Vincent MJ, Bergeron E, Benjannet S, Erickson BR, Rollin PE, et al. (2005) Chloroquine is a potent inhibitor of SARS coronavirus infection and spread. Virol J 2: 69.

19. Touret F, de Lamballerie X (2020) Of chloroquine and COVID-19. Antiviral Research 177: 104762.

20. Keyaerts E, Li S, Vijgen L, Evelien Rysman, Jannick Verbeeck, et al. (2009) Antiviral Activity of Chloroquine against Human Coronavirus OC43 Infection in Newborn Mice. Antimicrobial Agents and Chemotherapy 53: 3416-3421.

21. Tan YW, Yam WK, Sun J, Chu JJH (2018) An evaluation of Chloroquine as a broad-acting antiviral against Hand, Foot and Mouth Disease. Antiviral Res 149: 143-149.

22. Yan Y, Zou Z, Sun Y, Xiao Li, Kai Feng Xu, et al. (2013) Anti-malaria drug chloroquine is highly effective in treating avian influenza A H5N1 virus infection in an animal model. Cell Research 23(2): 300-302.

23. Paton NI, Lee L, Xu Y, Ooi EE, Cheung YB, et al. (2011) Chloroquine for influenza prevention: a randomised, double-blind, placebo controlled trial. The Lancet Infectious Diseases 11(9): 677-683.

24. Shimizu Y, Yamamoto S, Homma M, Ishida N (1972) Effect of chloroquine on the growth of animal viruses. Archiv für die gesamte Virusforschung 36(1): 93-104.

25. Keyaerts E, Vijgen L, Maes P, Neyts J, Van Ranst M (2004) In vitro inhibition of severe acute respiratory syndrome coronavirus by chloroquine. Biochemical and biophysical research communications 323(1): 264-268.

26. Inglot AD (1969) Comparison of the Antiviral Activity in vitro of some Non-steroidal Anti-inflammatory Drugs. Journal of General Virology 4(2): 203-214.

27. Hoffmann M, Kleine-Weber H, Schroeder S, Krüger N, Herrler T, et al (2020) SARS-CoV-2 Cell Entry Depends on ACE2 and TMPRSS2 and Is Blocked by a Clinically Proven Protease Inhibitor. Cell S0092-8674(20): 30229-30234.

28. (2009) FOIPAN (Camostat mesilate) Commodity Classification of Japan.

29. (2020) Camostat. Drugs.

30. Lucas JM, Heinlein C, Kim T, Hernandez SA, Malik MS, et al. (2014) The androgen-regulated protease TMPRSS2 activates a proteolytic cascade involving components of the tumor microenvironment and promotes prostate cancer metastasis. Cancer discovery 4(11): 1310-1325. 
31. Chang CC, Cheng AC, Chang AB (2014) Over-the-counter (OTC) medications to reduce cough as an adjunct to antibiotics for acute pneumonia in children and adults. The Cochrane database of systematic reviews 2007:Cd006088.

32. Danelius E, Andersson H, Jarvoll P, Lood K, Gräfenstein J, Erdélyi M (2017) Halogen Bonding: A Powerful Tool for Modulation of Peptide Conformation. Biochemistry 56: 3265-3272.

33. Han S, Mallampalli RK (2015) The Role of Surfactant in Lung Disease and Host Defense against Pulmonary Infections. Ann Am Thorac Soc 12(5): 765-774.
34. Plomer M, de Zeeuw J (2017) [More than expectorant: new scientific data on ambroxol in the context of the treatment of bronchopulmonary diseases]. MMW Fortschritte der Medizin 159(Suppl): 22-33.

35. Gao X, Huang Y, Han Y, Bai CX, Wang G (2011) The protective effects of Ambroxol in Pseudomonas aeruginosa-induced pneumonia in rats. Arch Med Sci 7(3): 405-413.

36. Laporte M, Naesens L (2017) Airway proteases: an emerging drug target for influenza and other respiratory virus infections. Current opinion in virology 24: 16-24. 